\title{
Attacking Boron Nucleophiles: NMR Properties of 5-membered Diazaborole Rings
}

\author{
Janet E. Del Bene, José Elguero, Ibon \\ Alkorta, Manuel Yáñez, and Otilia Mó
}

\section{Supporting Information}

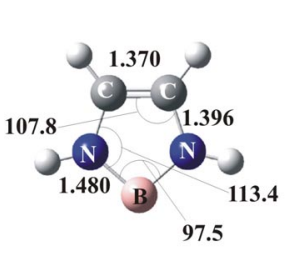

2

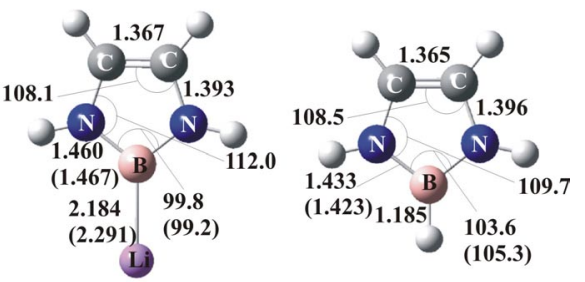

Optimized MP2/6-311++G(d,p) structures of diazaborole anion (2), Lidiazaborole (3), and diazaborole (4). Bond lengths are in $\AA$ and bond angles in degrees. Experimental solid-state values from Ref. 1a in parentheses. The molecules studied experimentally have 2,6-diisopropylphenyl groups bonded to the two nitrogens.

One-and two-bond spin-spin coupling data $(\mathrm{Hz})$ for 6-membered boron rings ${ }^{\mathrm{a}}$

\begin{tabular}{|c|c|c|c|c|}
\hline \multirow[b]{2}{*}{ One-bond } & \multirow{2}{*}{$\begin{array}{c}\text { Borazine anion }(6)^{\mathrm{b}} \\
\text { FC }\end{array}$} & \multirow{2}{*}{$\begin{array}{c}\text { Li-borazine }(7)^{\mathrm{c}} \\
\text { FC }\end{array}$} & \multicolumn{2}{|c|}{ Borazine $(8)^{d}$} \\
\hline & & & $\mathrm{FC}$ & $\mathrm{J}$ \\
\hline $\mathrm{B}_{1}-\mathrm{N}_{2}$ & -9.7 & -17.4 & -28.7 & -26.7 \\
\hline $\mathrm{B}_{1}-\mathrm{X}^{\mathrm{e}}$ & ----- & 140.4131 .2 & ----- & \\
\hline
\end{tabular}

a) Date for borazine and Li-borazine taken from ref. 2 .

b) $\mathrm{C}_{2 \mathrm{v}}$ symmetry with the negative charge on $\mathrm{B}_{1}$.

c) $\mathrm{C}_{2 \mathrm{v}}$ symmetry with $\mathrm{Li}$ at $\mathrm{B}_{1}$.

d) $\mathrm{D}_{3 \mathrm{~h}}$ symmetry.

e) $\mathrm{X}=\mathrm{Li}$ for Li-borazine and $\mathrm{H}$ borazine.

\section{REFERENCES}

3. Gaussian 03, Frisch, M. J.; Trucks, G. W.; Schlegel, H. B.; Scuseria, G.

E.; Robb, M. A.; Cheeseman, J. R.; Montgomery, Jr., J. A.; Vreven, T.; Kudin, K. N.; Burant, J. C.; Millam, J. M.; Iyengar, S. S.; Tomasi, J.; Barone, V.; Mennucci, B.; Cossi, M.; Scalmani, G.; Rega, N.; Petersson,

G. A.; Nakatsuji, H.; Hada, M.; Ehara, M.; Toyota, K.; Fukuda, R.; Hasegawa, J.; Ishida, M.; Nakajima, T.; Honda, Y.; Kitao, O.; Nakai, H.; Klene, M.; Li, X.; Knox, J. E.; Hratchian, H. P.; Cross, J. B.; Adamo, C.; Jaramillo, J.; Gomperts, R.; Stratmann, R. E.; Yazyev, O.; Austin, A. J.; Cammi, R.; Pomelli, C.; Ochterski, J. W.; Ayala, P. Y.; Morokuma, K.; Voth, G. A.; Salvador, P.; Dannenberg, J. J.; Zakrzewski, V. G.; Dapprich, S.; Daniels, A. D.; Strain, M. C.; Farkas, O.; Malick, D. K.; Rabuck, A. D.; Raghavachari, K.; Foresman, J. B.; Ortiz, J. V.; Cui, Q.; Baboul, A. G.; Clifford, S.; Cioslowski, J.; Stefanov, B. B.; Liu, G.;

Liashenko, A.; Piskorz, P.; Komaromi, I.; Martin, R. L.; Fox, D. J.;

Keith, T.; Al-Laham, M. A.; Peng, C. Y.; Nanayakkara, A.; Challacombe, M.; Gill, P. M. W.; Johnson, B.; Chen, W.; Wong, M. W.; Gonzalez, C. and Pople, J. A. Gaussian, Inc., Pittsburgh PA, 2003.

7. ACES II is a program product of the Quantum Theory Project, University of Florida. Stanton, J. F.; Gauss, J.; Perera, S. A.; Watts, J. D.; Yau, A D.; Nooijen, M.; Oliphant, N.; Szalay, P. G.; Lauderdale, W. J.; Gwaltney, S. R.; Beck, S.; Balková, A.; Bernholdt, D. E.; Baeck, K. K.; Rozyczko, P.; Sekino, H.; Huber, C.; Pittner, J.; Cencek, W.; Taylor, D.; Bartlett, R. J. Integral packages included are VMOL (Almlöf, J.; Taylor, P. R.); VPROPS (Taylor, P.); ABACUS (Helgaker, H.; Jensen, H. J. Aa.; Jørgensen, P.; Olsen, J.; Taylor, P. R.); HONDO/GAMESS (Schmidt, M. W.; Baldridge, K. K.; Boatz, J. A.; Elbert, S. T.; Gordon, M. S.; Jensen, J. J.; Koseki, S.; Matsunaga, N.; Nguyen, K. A.; Su, S.; Windus, T. L.; Dupuis, M.; Montgomery, J. A.). 
